\title{
A Dynamic Short-Turning Bus Control for Uncertain Demand
}

\author{
Hu Zhang, Shuzhi Zhao, Huasheng Liu, and Jin Li \\ College of Transportation, Jilin University, Changchun 130022, China \\ Correspondence should be addressed to Jin Li; li_jin@jlu.edu.cn
}

Received 28 June 2017; Revised 21 October 2017; Accepted 14 November 2017; Published 31 December 2017

Academic Editor: Luca D’Acierno

Copyright (C) $2017 \mathrm{Hu}$ Zhang et al. This is an open access article distributed under the Creative Commons Attribution License, which permits unrestricted use, distribution, and reproduction in any medium, provided the original work is properly cited.

\begin{abstract}
This paper formulates a dynamic approach for real-time bus control in uncertain demand. This dynamic approach aims to save the total cost for passengers and operators, while improving transit service reliability. An unfixed rolling horizon was implemented to choose the best dynamic approach. Real-time control predicts two discrete variables (arrival time and bus position) and determines the space-time point of buses. Furthermore, controlled actions include stop skipping and bus holding. The holding time starts when a bus serves a station and depends on previous intervals of passenger boarding and alighting at the station. The stop skipping action allows a bus to skip not only stations with a short-turning exception, but also stations with low demand for boarding that have been alighted in the short-turning segment. Stop skipping and bus holding actions for short-turning service both decrease the travel time of served passengers and the running time of buses, thus improving transit service reliability. A genetic algorithm was applied to solve the problem and the validity of the proposed dynamic approach was tested with four different scenarios. The result of these tests shows that a dynamic short-term bus control can significantly reduce total cost and improve transit service reliability.
\end{abstract}

\section{Introduction}

In a transit system that is faced with a certain demand of passengers, a short-turning strategy is usually applied to meet the unbalanced demand and to reduce the running time of buses. A bus can serve a segment with high demand; however, demand is uncertain during operation. The random arrival of passengers at stations along the bus route results in an uncertain number of boarding and alighting passengers. Then, buses may spend different serving durations at the same station. The arrival time of buses at stations cannot be predicted, which will create bus bunching or even overtaking. Transit service reliability may be decreased, thus increasing the travel time of passengers. Therefore, a dynamic approach should be implemented to guarantee transit service reliability and to save the cost of a transit system.

Furth [1] and Ceder [2] studied the departure frequency of vehicles with short-turning strategies and found a linear relationship with the frequency with which vehicles served all stations. Delle Site and Filippi [3], Ulusoy et al. [4], Cortés et al. [5], and Zhang et al. [6] aimed to decrease the total cost and to determine the turn-back station as well as the frequency of vehicles.
With regard to the dynamic strategy for such uncertain factors, ITS technologies are typically utilized to provide vehicle movements and passenger flows. Examples are the automatic vehicle location (AVL), automatic vehicle identification (AVI) systems, and automatic passenger counters (APC). Turnquist and Blume [7] and Abkowitz and Lepofsky [8] developed a holding strategy to increase service regularity. Yu et al. [9] predicted the subsequent transit service after a fixed rolling horizon based on transit service reliability assessment and presented a real-time partway-deadheading strategy. Chen et al. [10] considered the stochastic travel time and determined station skipping under a fixed rolling horizon. Delgado et al. [11] proposed an integrated holding and boarding limit strategy that does not allow passengers to board buses once a strategic limit has been reached. Based on uncertain demand, Cortés et al. [12] and Sáez et al. [13] developed a fixed rolling horizon to present a hybrid predictive control for the real-time optimization of public transport systems.

These previous studies presented different controlled strategies to reduce the cost of a transit system, while improving the transit service. Furthermore, under a fixed rolling 


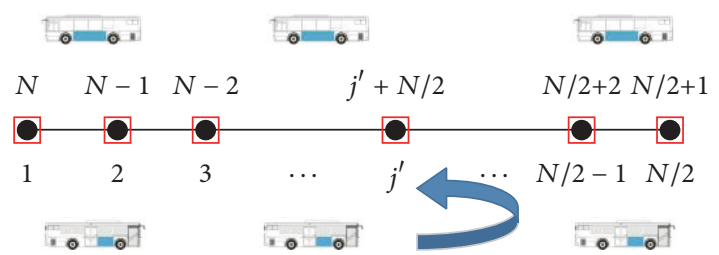

FIGURE 1: The dynamic short-turning strategy.

horizon, stop skipping and bus holding are used to balance the headway. This present study considered short-turning strategy patterns and developed an unfixed rolling horizon to meet the uncertain demand of passengers. Stop skipping and bus holding were also used to increase the transit service reliability for this study; however, there are differences from previous studies. To ensure uniform waiting time of passengers at the same station, dynamic actions equalize the serving time at the same station, which is different from the headway between consecutive vehicles. An objective function was formulated to minimize the total cost of the transit system and consequently a controlled scheme of stop skipping and bus holding could be determined. After this introduction, Section 2 describes the dynamic controlled strategy and describes the continuous prediction of the arrival time at different stations and positions. Section 3 establishes the objective function to minimize the total cost of the transit system. Based on genetic algorithms, Section 4 presents our solution methods to solve the objective function. Section 5 tests the dynamic strategy, compares our results to other strategies, and evaluates the performance for improving transit service reliability. Finally, Section 6 presents our conclusions.

\section{Problem Setting}

2.1. Dynamic Strategy Description. We assumed that buses operate dynamically on a bus route with $N$ stops. According to a given schedule, buses dispatch from station 1 , arrive at station $N / 2$ in one direction, and then operate from station $N / 2+1$ to station $N$ in the opposite direction. As shown in Figure 1, buses with an optimized strategy will turn back at station $j^{\prime}\left(j^{\prime}+N / 2\right)$. We assumed the bus route to be divided into two parts: a segment with high demand and another segment with low demand, with the turn-back station $j^{\prime}\left(j^{\prime}+\right.$ $N / 2$ ) as the cut-off point between both segments. Furthermore, the segment with high demand is close to station $1(N)$ in both directions.

Under the short-turning strategy, and to deal with the uncertain demand at stations along the bus route, stop skipping and bus holding actions are implemented to improve transit service reliability. The stop skipping strategy allows buses to skip a station, consequently focusing on serving a station with high demand and increasing the speed of buses. The bus holding strategy requires buses to wait at a serving station for a specific amount of time, which can increase the headway to preceding vehicles and avoid bunching or overtaking. A bus holding strategy corresponds to the stop skipping action. If a bus skips a station, the holding action cannot be triggered. Furthermore, if a bus is selected to serve a particular station, the holding time of buses will be calculated again.

In a dynamic approach, the index of buses is denoted as $i$ and the index of stations is denoted as $j$. And the arrival time of bus $i$ at station $j$ is related to the departure time from station $j-1$, the running time, and the acceleration and deceleration time of buses, which is affected by the stop skipping and bus holding. If the stop skipping is triggered, the acceleration and deceleration time of buses is equal to zero. Therefore, there are two discrete variables to predict: the arrival time of bus $i$ at station $j$ (denoted as $\widehat{T}_{i, j}^{A}$ ) and the position of bus $i$ (denoted as $l_{i}(t)$ ).

The rolling horizon of the dynamic control is defined as $M \in\left\{m_{1}, m_{2}, \ldots, m_{k}\right\}$, where $m_{k}$ represents the number of buses within a rolling horizon. To avoid an excessive number of passengers who will be skipped and excess buses capacity, the rolling horizon remains unfixed to maximize the effect of a short-turning strategy, thus allowing more buses to serve the bus route, using a short-turning strategy. The rolling horizon corresponds to the lowest demand of a station along the bus route. We assumed that buses $i, i+1, \ldots$, and $i+m_{k}-1$, which are controlled, may turn back before the end of the bus route. The period of the $k$ th rolling horizon $\widehat{T}_{k}(t)$ and the number of buses under the $k$ th rolling horizon $m_{k}$ can then be calculated by

$$
\begin{aligned}
\widehat{T}_{k}(t) & =\sum^{m_{k}} \bar{h} \\
0 & <Q_{c}-\sum^{m_{k}} \bar{h} \cdot \min _{j=1,2, \ldots, N}\left[\hat{\lambda}_{j}\right]<\bar{h} \cdot \min _{j=1,2, \ldots, N}\left[\widehat{\lambda}_{j}\right],
\end{aligned}
$$

where $\bar{h}$ represents the average departure interval, $Q_{c}$ represents the maximum load of bus, and $\hat{\lambda}_{j}$ represents the demand of passengers at stations. The rolling horizon $m_{k}$ can be determined via (2).

2.2. Short-Turning Strategy without Stop Skipping or Bus Holding. When implementing a short-turning strategy, buses operating along a segment of the bus route with high demand will turn back before a segment with low demand. Without stop skipping or bus holding actions, the arrival time of bus $i$ at station $j T_{i, j}^{A}$ can be computed as

$$
T_{i, j}^{A}=T_{i, j-1}^{D}+t_{\mathrm{ac}}+t_{j-1, j}^{R}+t_{\mathrm{de}},
$$

where $T_{i, j-1}^{D}$ represents the departure time once bus $i$ departs from station $j-1$ and $t_{j-1, j}^{R}$ represents the running time between station $j-1$ and station $j . t_{\mathrm{ac}}$ represents the acceleration time of buses and $t_{\mathrm{de}}$ represents the deceleration time of buses, which can be obtained from the practical investigation for the startup acceleration and the deceleration velocity. According to the survey, $t_{\mathrm{de}}$ is set as $9 \mathrm{~s}$ and $t_{\mathrm{ac}}$ is set as $10 \mathrm{~s}$. When turn-back action is triggered at the turn-back station $j^{\prime}$, buses require the running time $t_{j^{\prime}, j^{\prime}+N / 2}^{R}$ to arrive 
at the opposite station $j^{\prime}+N / 2$ at the other direction and the arrival time of bus $i$ at station $j^{\prime}+N / 2$ is calculated as

$$
T_{i, j^{\prime}+N / 2}^{A}=T_{i, j^{\prime}}^{D}+t_{j^{\prime}, j^{\prime}+N / 2}^{R} .
$$

When buses arrive at a station, passengers can board and alight from the vehicle. The dwelling time of bus $i$ at station $j$ is denoted as $t_{i, j}^{E}$. The departure time $T_{i, j}^{D}$ can be determined via

$$
T_{i, j}^{D}=T_{i, j}^{A}+t_{i, j}^{E} .
$$

This above strategy is suitable for a specific passenger demand and when buses are able to operate according to schedule. With uncertain demand, the number of passengers at stations is subject to dynamic change, which cannot guarantee that buses arrive at stations on schedule. Therefore, a short-turning control strategy with stop skipping and bus holding is proposed to improve service reliability of segments of high and low demand.

\subsection{Short-Turn Control Strategy with Stop Skipping and} Bus Holding. The short-turning control strategy with stop skipping and bus holding describes the dynamic behavior of bus operation as a function of control actions. During the short-turning service, buses will utilize two control actions as inputs for the dynamic model, which is denoted as follows:

$$
\left\{\begin{array}{cccccc}
\hat{t}_{i, 1}^{h} & \hat{t}_{i, 2}^{h} & \cdots & \hat{t}_{i, j}^{h} & \cdots & \widehat{t}_{i, j^{\prime}}^{h} \\
y_{i, 1} & y_{i, 2} & \cdots & y_{i, j} & \cdots & y_{i, j^{\prime}}
\end{array}\right\}, \quad \hat{t}_{i, j}^{h} \geq 0, \quad y_{i, j}=[0,1],
$$

where $y_{i, j}$ corresponds to the stop skipping action of bus $i$ at station $j$ and if the bus skips the station $y_{i, j}=0$; otherwise, $y_{i, j}=1 . \hat{t}_{i, j}^{h}$ represents the holding time of bus $i$ at station $j$ after passengers have boarded and alighted and the holding time appears only if buses dwell at a station. If the bus skips the station, the holding time is zero.

By using stop skipping and bus holding control actions, buses will be dynamically controlled, while they arrive at a station. The discrete arrival time of such a dynamic control strategy once bus $i$ arrives at station $j$ can be computed as

$$
\widehat{T}_{i, j}^{A}=\widehat{T}_{i, j-1}^{D}+t_{\mathrm{ac}} \cdot y_{i, j-1}+t_{j-1, j}^{R}+t_{\mathrm{de}} \cdot y_{i, j} .
$$

The turn-back station $j^{\prime}$ is the terminal point, where buses are determined to serve in one direction. The arrival time at station $j^{\prime}+N / 2$ from station $j^{\prime}$ uses a similar strategy to that without dynamic actions, which is described as

$$
\widehat{T}_{i, j^{\prime}+N / 2}^{A}=\widehat{T}_{i, j^{\prime}}^{D}+t_{j^{\prime}, j^{\prime}+N / 2}^{R} .
$$

In the control strategy proposed here, both dynamic actions have a strong influence on the dwelling time at stations. The dwelling time includes the bus service time and the holding time. The discrete viable $y_{i, j}$ determined whether bus $i$ will serve station $j$ and then the holding time $\widehat{t}_{i, j}^{h}$ can be computed. Therefore, the departure time will be updated as

$$
\widehat{T}_{i, j}^{D}=\widehat{T}_{i, j}^{A}+\left(\hat{t}_{i, j}^{E}+\widehat{t}_{i, j}^{h}\right) \cdot y_{i, j},
$$

where $\hat{t}_{i, j}^{E}$ represents the serving time of bus $i$ at station $j \cdot \hat{t}_{i, j}^{h}$ is used to improve transit service reliability and to prevent bus bunching. Due to the uncertain demand and the stop skipping strategy, the estimated arrival and departure times change dynamically and the headway of buses is difficult to retain consistent. To a certain extent, implementing bus holding can prevent bus bunching and overtaking.

The influence of skip-stop actions on passenger demand at stations is considered. Generally, the boarding passenger demand at a station can be calculated as the headway multiplied by the arrival rate of passengers. However, in our study, the current headway, denoted as $\widehat{H}_{i, j}$, is not the mean interval between consecutive vehicles $\left(\widehat{T}_{i, j}^{D}-\widehat{T}_{i-1, j}^{D}\right)$, as some previous studies have mentioned. Furthermore, the current headway is the interval of buses that do not skip the station. If bus $i-1$ (ahead) is determined to skip station $j$ and bus $i-2$ serves station $j$, the current headway $\widehat{H}_{i, j}$ is equal to $\widehat{T}_{i, j}^{D}-\widehat{T}_{i-2, j}^{D}$, rather than $\widehat{T}_{i, j}^{D}-\widehat{T}_{i-1, j}^{D}$. The current headway $\widehat{H}_{i, j}$ can thus be computed as

$$
\begin{aligned}
& \widehat{H}_{i, j} \\
& = \begin{cases}\widehat{T}_{i, j}^{D}-\widehat{T}_{i-1, j}^{D} & y_{i-1, j}=1 \\
\widehat{T}_{i, j}^{D}-\widehat{T}_{i-2, j}^{D} & y_{i-1, j}=0, y_{i-2, j}=1 \\
\widehat{T}_{i, j}^{D}-\widehat{T}_{i-3, j}^{D} & y_{i-1, j}+y_{i-2, j}=0, \quad y_{i-3, j}=1 \\
\vdots & \\
\widehat{T}_{i, j}^{D}-\widehat{T}_{i-k, j}^{D} & y_{i-1, j}+y_{i-2, j}+\cdots+y_{i-k+1, j}=0, \quad y_{i-k, j}=1 .\end{cases}
\end{aligned}
$$

The proposed controlled strategy was implemented to equalize the intervals of buses where passengers can board and alight at one station and to improve the degree of passenger satisfaction, enabling passengers at one station to experience equal waiting times. The holding time can be computed as

$$
\widehat{t}_{i, j}^{h}= \begin{cases}\widehat{H}_{i-1, j}-\widehat{H}_{i, j} & \text { if } \widehat{H}_{i-1, j}>\widehat{H}_{i, j}, y_{i, j}=1 \\ 0 & \text { otherwise. }\end{cases}
$$

Regarding the strategy of buses at the turn-back station, we determined the position of the previous bus and the next bus from the turn-back bus, as the bus conducts the turnback. Therefore, the position of the predicted bus $i$ along the route at constant $t, \widehat{l}_{i}(t)$, can be described as a function of the 
instantaneous speed $\widehat{v}_{i}(t)$ of the bus. The position of a bus $i$ can be computed as follows:

$$
\widehat{l}_{i}(t)= \begin{cases}0+\int_{0}^{t} \widehat{v}_{i}(t) d t & t \leq \widehat{T}_{i, j^{\prime}}^{D} \\ \int_{0}^{\widehat{T}_{i, j^{\prime}}^{D}} \widehat{v}_{i}(t) d t-\int_{\widehat{T}_{i, j^{\prime}}^{D}}^{t} \widehat{v}_{i}(t) d t & t>\widehat{T}_{i, j^{\prime}}^{D} .\end{cases}
$$

The speed $\widehat{v}_{i}(t)$ depends on the continuous time and applied control actions and can be described as

$$
\begin{aligned}
& \widehat{v}_{i}(t) \\
& = \begin{cases}\mathrm{ac} \cdot t & \widehat{T}_{i, j}^{D} \leq t \leq \widehat{T}_{i, j}^{D}+t_{\mathrm{ac}} \cdot y_{i, j} \\
\bar{v} & \widehat{T}_{i, j}^{D}+t_{\mathrm{ac}} \cdot y_{i, j} \leq t \leq \widehat{T}_{i, j}^{D}+t_{\mathrm{ac}} \cdot y_{i, j}+t_{j, j+1}^{R} \\
\mathrm{de} \cdot t & \widehat{T}_{i, j}^{D}+t_{\mathrm{ac}} \cdot y_{i, j}+t_{j, j+1}^{R} \leq t \leq \widehat{T}_{i, j+1}^{A} \\
0 & \widehat{T}_{i, j+1}^{A}<t<\widehat{T}_{i, j+1}^{D} .\end{cases}
\end{aligned}
$$

The position of the bus $i^{\prime}$ previous to the turn-back bus at station $j^{\prime}\left(j^{\prime}+N / 2\right)$ is close to the position of the turnback bus $i$. Under the continuous time $t$, the position of the previous bus $i^{\prime}$ can be determined via

$$
\widehat{l}_{i}\left(t_{i, j^{\prime}}^{D}\right)-\widehat{l}_{i^{\prime}}\left(t_{i, j^{\prime}}^{D}\right)=\min _{l=i-1, i-2, \ldots}\left[\widehat{l}_{i}\left(t_{i, j^{\prime}}^{D}\right)-\widehat{l}_{l}\left(t_{i, j^{\prime}}^{D}\right)\right]
$$

Then, the headway $\widehat{H}_{i, j^{\prime}}$ between bus $i$ and bus $i^{\prime}$ can be updated and the holding time of bus $i$ at the turn-back station $j^{\prime}$ can be calculated via (11).

The serving time $t_{i, j}^{E}$ depends on the boarding and alighting behaviors of passengers who select the bus that serves their origin and destination. The serving time $t_{i, j}^{E}$ and the number of passengers boarding and alighting at station $j$ can be expressed via

$$
\begin{aligned}
t_{i, j}^{E} & =\max \left[b \cdot \widehat{N}_{i, j}^{+}, a \cdot \widehat{N}_{i, j}^{-}\right] \\
\widehat{N}_{i, j}^{+} & =\widehat{H}_{i, j} \sum_{k=j+1}^{N / 2} \widehat{\lambda}_{i, j \rightarrow k} \cdot y_{i, j} \cdot y_{i, k} \\
\widehat{N}_{i, j}^{h+} & =\widehat{t}_{i, j}^{h} \sum_{k=j+1}^{N / 2} \widehat{\lambda}_{i, j \rightarrow k} \cdot y_{i, j} \cdot y_{i, k} \\
\widehat{N}_{i, j}^{-} & =\sum_{k=1}^{j-1}\left[\widehat{\lambda}_{i, k \rightarrow j} \cdot y_{i, k} \cdot y_{i, j} \cdot\left(\widehat{H}_{i, k}+\widehat{t}_{i, k}^{h}\right)\right],
\end{aligned}
$$

where $\widehat{N}_{i, j}^{+}$represents the number of passengers who board bus $i$ at station $j$ after waiting. $\widehat{N}_{i, j}^{-}$represents the number of passengers alighting from bus $i$ at station $j . \widehat{N}_{i, j}^{h+}$ represents the number of passengers boarding bus $i$ at station $j$ during holding time. $\widehat{\lambda}_{i, j \rightarrow k}$ represents the demand of passengers, served by bus $i$, who travel from station $j$ to station $k$. Variables $b$ and $a$ represent the passenger boarding and alighting time per passenger, respectively.

Beyond the predicted variables $\widehat{T}_{i, j}^{A}$ and $\widehat{l}_{i}(t)$, this study considered the passenger load between adjacent stations, denoted as $\widehat{L}_{i, j}$, to compute the in-vehicle time of passengers during their trips. The variable $\widehat{L}_{i, j}$ can be expressed by

$$
\widehat{L}_{i, j}= \begin{cases}\widehat{L}_{i, j-1}+\left(\widehat{N}_{i, j}^{+}+\widehat{N}_{i, j}^{h+}-\widehat{N}_{i, j}^{-}\right) \cdot y_{i, j} & j \geq 2 \\ 0 & j=1 .\end{cases}
$$

Furthermore, when the $k$ th rolling horizon is over, the passenger demand that remains at the station will be fed back to the $k+1$ th rolling horizon. The passenger demand that remains at the station from the $k$ th rolling horizon under the optimization scheme will be updated as

$$
\widehat{N}_{m_{k}, j}^{+}=\widehat{H}_{m_{k}, j} \sum_{k=j+1}^{N / 2} \widehat{\lambda}_{i, j \rightarrow k} \cdot y_{i, j} \cdot y_{i, k},
$$

where $\widehat{H}_{m_{k}, j}$ represents the intervals between the last bus $m_{k}$ and the bus ahead. If the last bus $m_{k}$ does not skip the station, the value of $\widehat{H}_{m_{k}, j}$ is equal to zero. $\widehat{H}_{m_{k}, j}$ can be expressed as

$$
\widehat{H}_{m_{k}, j}= \begin{cases}0 & y_{m_{k}, j}=1 \\ \widehat{T}_{m_{k}, j}^{D}-\widehat{T}_{m_{k}-1, j}^{D} & y_{m_{k}, j}=0, \quad y_{m_{k}-1, j}=1 \\ \widehat{T}_{m_{k}, j}^{D}-\widehat{T}_{m_{k}-2, j}^{D} & y_{m_{k}, j}=1, \quad y_{m_{k}-1, j}=0, \quad y_{m_{k}-2, j}=1 \\ \widehat{T}_{m_{k}, j}^{D}-\widehat{T}_{m_{k}-3, j}^{D} & y_{m_{k}, j}=1, \quad y_{m_{k}-1, j}+y_{m_{k}-2, j}=0, \quad y_{m_{k}-3, j}=1 \\ \vdots & \\ \widehat{T}_{m_{k}, j}^{D}-\widehat{T}_{m_{k}-l, j}^{D} & y_{m_{k}, j}=1, \quad y_{m_{k}-1, j}+y_{m_{k}-2, j}+\cdots+y_{m_{k}-l+1, j}=0, y_{m_{k}-l, j}=1 .\end{cases}
$$




\section{Optimization Model}

To make decisions in real-time, this part formulates an objective function that reduces the total cost of a dynamic system. The objective function comprises four components, oriented to costs of users and operators. Within a prediction horizon $m$, the objective function is formulated by

$$
\begin{aligned}
& \min C=\sum_{i}^{m_{k}}\left\{c_{1} \sum_{j=2}^{N}\left(\widehat{T}_{i, j}^{D}-\widehat{T}_{i, j-1}^{D}\right)\right. \\
& +c_{2} \sum_{j=1}^{N-1}\left(\widehat{T}_{i, j}^{D}-\widehat{T}_{i-1, j}^{D}\right)^{2} \cdot \sum_{k=j+1}^{N} \widehat{\lambda}_{i, j \rightarrow k} \cdot y_{i, j} \cdot y_{i, k} \\
& +c_{3} \sum_{j=1}^{N-1}\left(t_{j, j+1}^{R}+\widehat{t}_{i, j}^{E} \cdot y_{i, j}\right) \cdot \widehat{L}_{i, j}+c_{4} \sum_{j=1}^{N-1} \widehat{t}_{i, j}^{h} \cdot \widehat{L}_{i, j} \\
& +c_{5} \sum_{j=1}^{N-1}\left[\widehat{H}_{i, j}-\left(\widehat{T}_{i, j}^{D}-\widehat{T}_{i-1, j}^{D}\right)\right]^{2} \\
& \left.\cdot \sum_{k=j+1}^{N} \widehat{\lambda}_{i, j \rightarrow k} \cdot y_{i, j} \cdot y_{i, k}\right\} .
\end{aligned}
$$

Here, the first term of (19) corresponds to the running time of buses and can reveal the performance of stop skipping strategy to operators. The second term quantifies the waiting time for passengers and is dependent on the intervals of buses where passengers can board and alight at one station, $\widehat{H}_{i, j}$. The third component measures the in-vehicle time of passengers during intervals between consecutive stations and their dwelling time. The fourth term is an additional holding time for passengers on load. The fifth component is a penalty for the extra waiting time of passengers who are skipped due to the stop skipping or short-turn strategy. $\widehat{H}_{i, j}-\left(\widehat{T}_{i, j}^{D}-\widehat{T}_{i-1, j}^{D}\right)$ represents the additional waiting time per passenger. The variables $c_{1}, c_{2}, c_{3}, c_{4}$, and $c_{5}$ are time values.

\section{Solution Method Based on Genetic Algorithms}

To solve the objective function provided in the previous sections, genetic algorithms were used to determine the turn-back station and the discrete scheme of stop skipping, which is a problem of nonlinear 0,1 . Genetic algorithms can efficiently cope with such mixed-integer, nonlinear problems. The corresponding holding time can be calculated with the initial scheme. In this study, the random arrival of passengers at stations reflects the actual situation because the arrival of passenger flow is not fixed. The random arrival of passengers will be dealt with by a Monte Carlo Simulation method.

The specific steps of solution method corresponding to Figure 2, which includes the Monte Carlo Simulation method and the feedback from the optimization problem, are as follows.
Step 1 (initialize the parameters). Set the number of iterations of the genetic algorithm $N_{G}$, the crossover probability $P_{c}$, and the mutation probability $P_{m}$. Then, generate initial skipping schemes $y_{i, j^{\prime}}$ and $y_{i, j}$.

Step 2. The Monte Carlo Simulation method is applied to deal with the random arrival of passengers and to compute both holding time and objective function.

Step 2.1 (set the counter of simulations $n_{\mathrm{mc}}$ ). Perform passenger demand sampling and generate the passenger demand based on its distribution function $\hat{\lambda}_{i, j}$. Furthermore, the passenger demand that remains at the station from the $k-1$ th rolling horizon $\widehat{N}_{m_{k-1}, j}^{+}$is superposed.

Step 2.2. Determine the number of the rolling horizons $\widehat{m}_{k}^{\left(n_{\mathrm{mc}}\right)}$ using (1)-(2).

Step 2.3. Update the value of parameters in the solution using (3)-(16), including bus arrival time, departure time, the position of a bus, headways, and the number of boarding as well as alighting passengers.

Step 2.4. Compute the holding time $\hat{t}_{i, j}^{h, n_{\mathrm{mc}}}$ based on (10). Calculate the value of the objective function $\widehat{C}^{\left(n_{\mathrm{mc}}\right)}$ based on (19).

The update of $\bar{m}_{k}^{\left(n_{\mathrm{mc}}\right)}, \bar{t}_{i, j}^{h, n_{\mathrm{mc}}}$, and $\bar{C}^{\left(n_{\mathrm{mc}}\right)}$ depends on the average value of the simulation value:

$$
\begin{aligned}
\bar{m}_{k}^{\left(n_{\mathrm{mc}}\right)}= & \frac{\widehat{m}_{k}^{\left(n_{\mathrm{mc}}\right)}+\left(n_{\mathrm{mc}}-1\right) \cdot \bar{m}_{k}^{\left(n_{\mathrm{mc}}-1\right)}}{n_{\mathrm{mc}}} \\
\bar{t}_{i, j}^{h, n_{\mathrm{mc}}} & =\frac{\widehat{t}_{i, j}^{h, n_{\mathrm{mc}}}+\left(n_{\mathrm{mc}}-1\right) \cdot \bar{t}_{i, j}^{h, n_{\mathrm{mc}}-1}}{n_{\mathrm{mc}}} \\
\bar{C}^{\left(n_{\mathrm{mc}}\right)} & =\frac{\widehat{C}^{\left(n_{\mathrm{mc}}\right)}+\left(n_{\mathrm{mc}}-1\right) \cdot \bar{C}^{\left(n_{\mathrm{mc}}-1\right)}}{n_{\mathrm{mc}}} .
\end{aligned}
$$

Step 2.5. If these are exceeding the maximal number of Monte Carlo simulations, then stop; otherwise, go to Step 2.1.

Step 3. Because the objective function is formed to minimize total costs, the fitness function is equal to the reciprocal of the objective function.

Step 4. The selection, crossover, and mutation of chromosomes are applied to produce new chromosomes. The crossover probability is set to 0.5 ; the mutation probability is set to 0.01 .

Step 5. An elitist preservation strategy is adopted to improve both search speed and search precision. A sample after Step 4 is replaced with the best sample before Step 4 .

Step 6. If these are exceeding the maximal number of generation, then stop; otherwise, go to Step 2. 


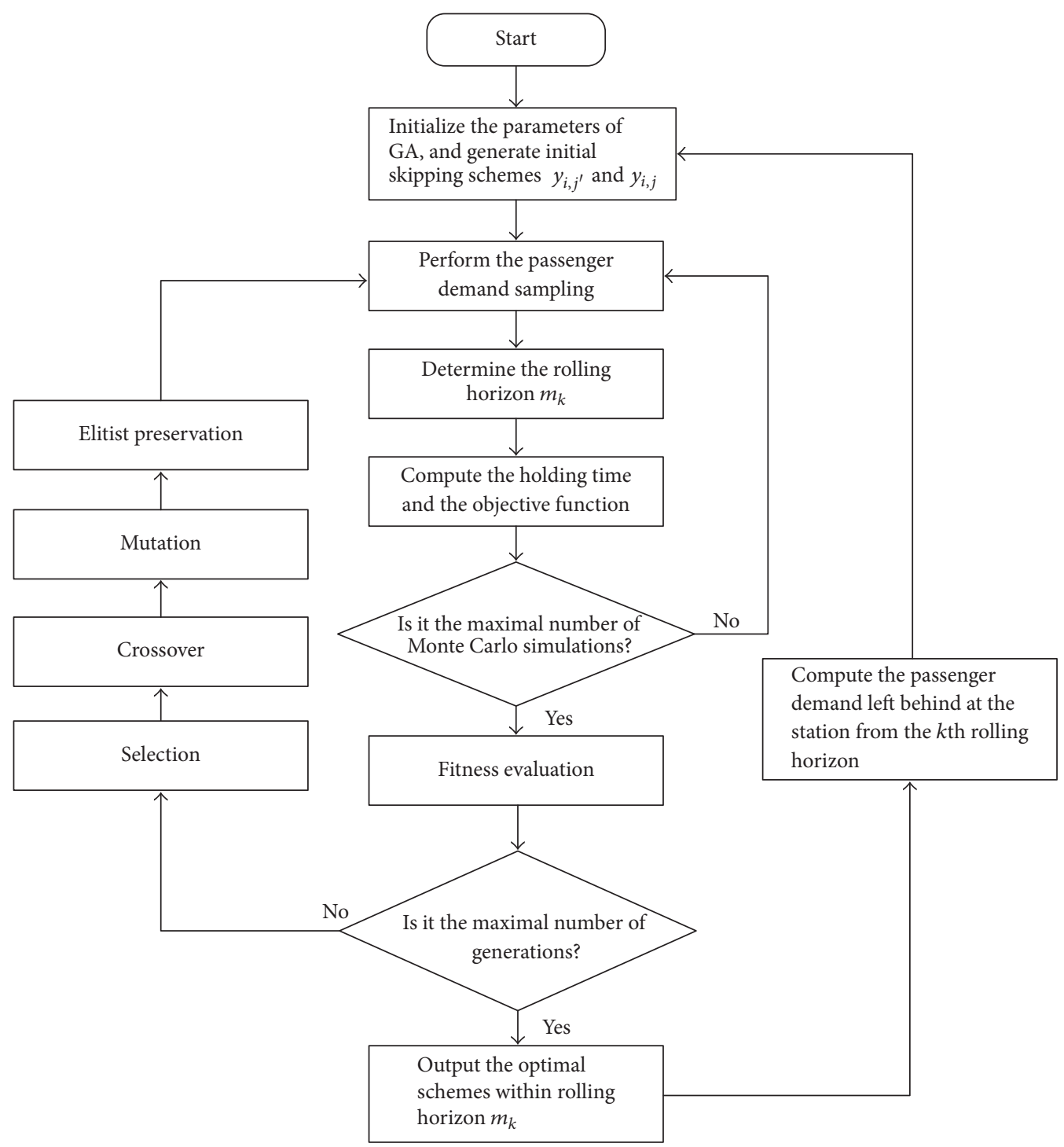

FIGURE 2: Solution method based on genetic algorithms.

Step 7. Output the optimization program during the $k$ th rolling horizon. Furthermore, compute the passenger demand that remains at the station from the $k$ th rolling horizon $\widehat{N}_{m_{k}, j}^{+}$; then, return to Step 1.

\section{Numerical Test}

The proposed dynamic approach was applied over a bus corridor and its basic data came from bus route number 6 of Changchun City in China. The bus corridor comprises 20 stations in each direction and includes a length of $9.9 \mathrm{~km}$. Without a dynamic approach, serving the entire route takes $29 \mathrm{~min}$. The departure interval is $4 \mathrm{~min}$. The average passenger demand is deduced from previous days, as shown in Figures 3 and 4, and follows a random distribution with demand rates differentiated by station and period. The variance of demand rates is set as $0.13 \lambda_{i, j}$. The turn-back station can be selected from stations $14,15,16$, and 20 . The speed of buses between stations is $19 \mathrm{~km} / \mathrm{h}$. The passenger boarding and alighting times are $5.2 \mathrm{~s} / \mathrm{pax}$ and $4.4 \mathrm{~s} / \mathrm{pax}$, respectively. The five time values $c_{1}, c_{2}, c_{3}, c_{4}$, and $c_{5}$ have been adjusted to $\$ 70 /$ veh-h, $\$ 14 /$ pax-h, $\$ 12 /$ pax-h, $\$ 9 /$ pax-h, and $\$ 15 /$ pax $-\mathrm{h}$, respectively, referring to the practicalities of everyday life and other studies $[10,14]$. The testing period was $2 \mathrm{~h}$ under the proposed dynamic approach and a warm-up period of $1 \mathrm{~h}$ was applied before the test.

In the solution method, the number of iterations of the genetic algorithm $N_{G}$ is set to 100 and the maximal counter of the Monte Carlo Simulation is 100. Furthermore, an elitist preservation strategy is adopted to improve the search speed and search precision, as shown in Step 5 of the solution method. Figure 5 depicts the convergence of the calculation with experimenting 10 times. The algorithm has good convergence and the optimal solutions of the objective function are found within 60 iterations. All processes were run on 


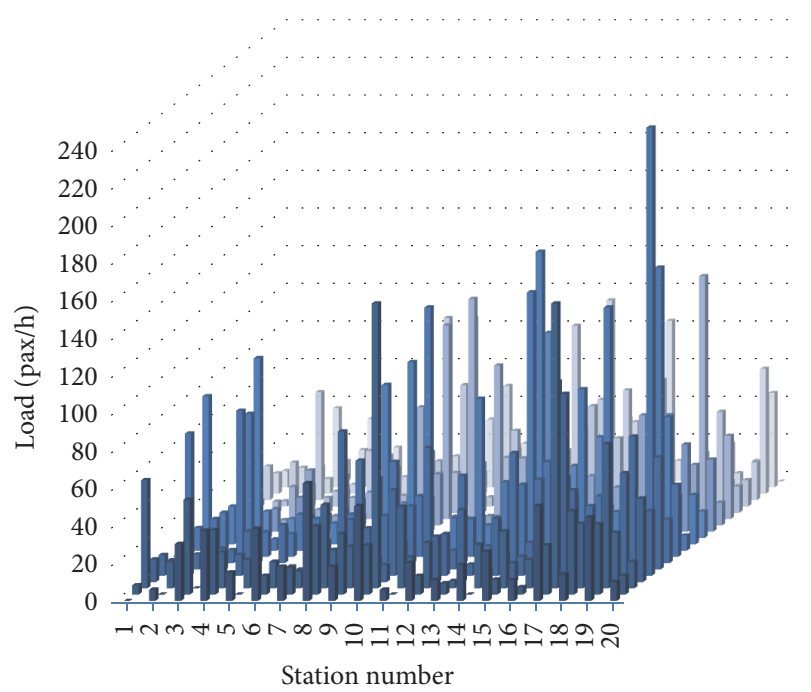

FIGURE 3: Average passenger demand in both directions during the early peak period.

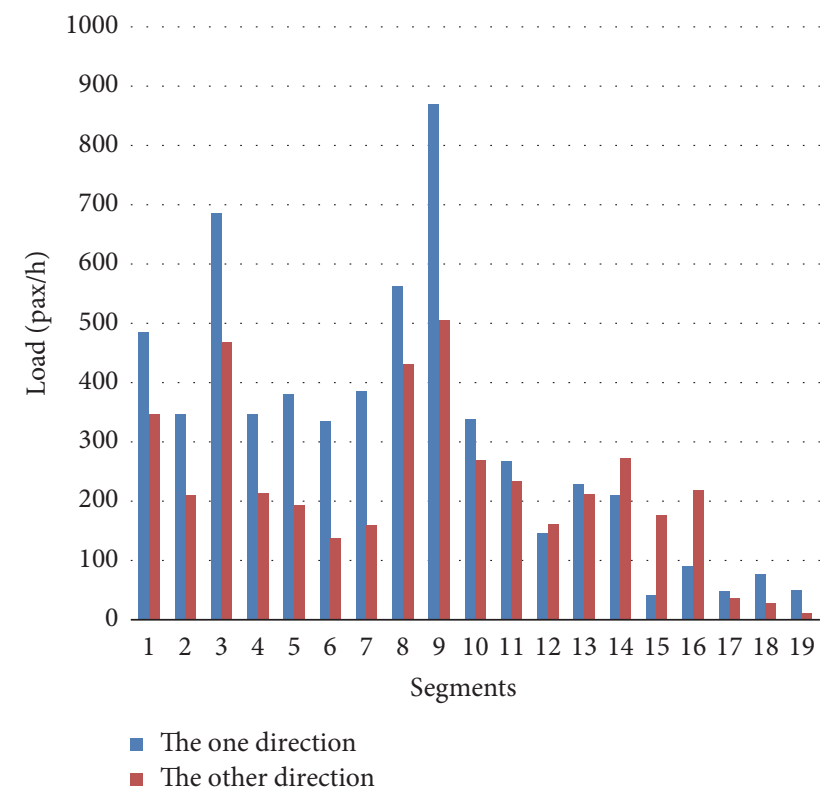

FIgURE 4: Passenger load between consecutive stations.

a Computer Intel Core I5, $2.4 \mathrm{GHz}$ with $8 \mathrm{~GB}$ RAM. Each optimization scheme could be obtained in less than $50 \mathrm{~s}$ and the departure interval of consecutive buses was $4 \mathrm{~min}$. Therefore, computing times can be ensured.

To test the validity of the proposed dynamic approach, four different strategies were tested: (1) all-stop (with no controls), (2) short-turning (without stop skipping or bus holding), (3) short-turning (with stop skipping but without bus holding), and (4) short-turning (with stop skipping and bus holding). The performances of four different strategies that use identical parameters have been evaluated. The first strategy was a traditional service in which buses need to stop

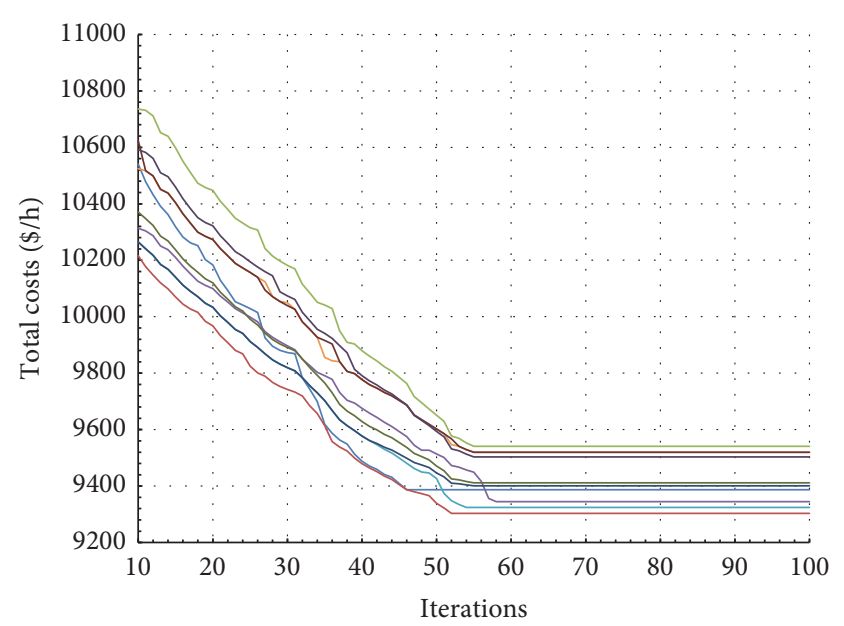

Figure 5: Result of each iteration.

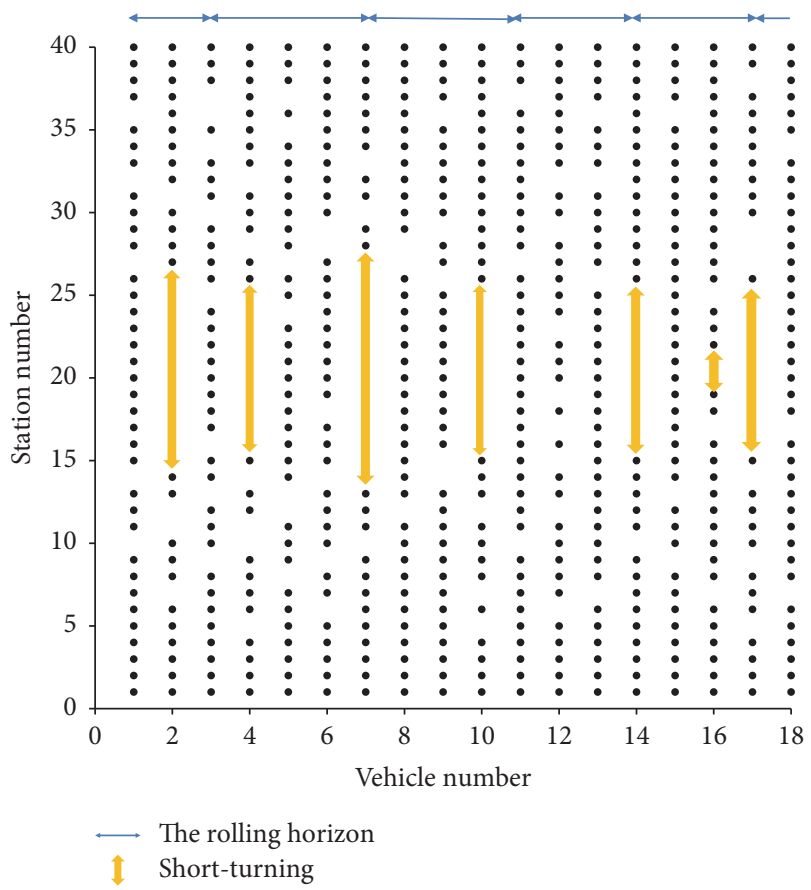

FIGURE 6: Optimal schemes with control strategy.

at every station and where each $y_{i, j}$ is equal to 1 . Only shortturning without the implementation of a stop skipping or bus holding strategy required the determination of the turnback station. Under short-turning with stop skipping, but without bus holding strategy, appropriate turn-back stations and respective stop skipping stations will be determined. Short-turning with stop skipping and bus holding requires the determination of turn-back stations and stop skipping stations and calculates the holding time at the serving station. The results are shown in Table 1 and present the total cost per hour under all four strategies. Figure 6 shows partial optimal output schemes of real-time control in the short-turning 
TABLE 1: Effects of the proposed dynamic approach.

\begin{tabular}{|c|c|c|c|c|c|c|c|}
\hline Control strategy & $\begin{array}{l}\text { Waiting } \\
\text { time cost }\end{array}$ & $\begin{array}{l}\text { In-vehicle } \\
\text { time cost }\end{array}$ & $\begin{array}{l}\text { Running } \\
\text { time cost }\end{array}$ & $\begin{array}{l}\text { Holding } \\
\text { time cost }\end{array}$ & $\begin{array}{c}\text { Penalty } \\
\text { cost }\end{array}$ & Total costs & $\begin{array}{l}\text { Reduction } \\
\text { in } \\
\text { weighted } \\
\text { total }\end{array}$ \\
\hline All-stop without controls & 932 & 6241 & 5128 & - & - & 12301 & - \\
\hline $\begin{array}{l}\text { Short-turning without stop } \\
\text { skipping or bus holding }\end{array}$ & 829 & 5984 & 4699 & - & - & 11512 & $6.41 \%$ \\
\hline $\begin{array}{l}\text { Short-turning with stop } \\
\text { skipping but without bus } \\
\text { holding }\end{array}$ & 603 & 5681 & 4306 & - & 197 & 10590 & $13.91 \%$ \\
\hline $\begin{array}{l}\text { Short-turning with stop } \\
\text { skipping and bus holding }\end{array}$ & 512 & 5127 & 4372 & 132 & 207 & 10350 & $15.86 \%$ \\
\hline
\end{tabular}

strategy with stop skipping and bus holding. The rolling horizon of the procedure is approximately 3-4 vehicles.

A previous study suggested that the short-turning strategy and stop skipping can reduce the running time of buses, while bus holding may delay buses, consequently improving the running time cost of buses and in-vehicle time cost of passengers. Via dynamic optimization of both strategies, the total cost of the transit system has been suggested to decrease, while the transit service reliability increases. The results of Table 1 show that three strategies that include short-turning can reduce the costs of users and operators that a traditional service (all-stop) creates. Furthermore, the holding strategy adds a holding time cost for passengers and increases the running time cost of buses; however, the negative effect of holding action has been compensated by incorporating a stop skipping strategy. The dynamic approach proposed here can reduce the total cost by $15.86 \%$, which results in an improved effect compared to short-turning without stop skipping or bus holding and the short-turning with stop skipping, but without bus holding.

To meet an uncertain passenger demand, the rolling horizon remains unfixed. Sensitivity to such an unfixed rolling horizon (as implemented in this study) was analyzed and compared to the performances of different fixed rolling horizon values and unfixed sizes (sizes $m_{k}=2, m_{k}=3, m_{k}=4$, and $m_{k}=5$, and unfixed $m_{k}$ ), as shown in Figure 7. Figure 7 shows that the unfixed rolling horizon results in the best effect of all dynamic approaches. With the unfixed rolling horizon, the total cost can be reduced in controlled cycles.

Finally, the effects of dynamic control actions (stop skipping and bus holding) on transit service reliability were analyzed. Figure 8 shows the interval of serving buses at the same station along the bus route and reveals that for the short-turn strategy the intervals between station 1 and station 15 were different than those between station 16 and station 20 . This is because buses were controlled to operate between station 1 and station 15 to serve a segment of high demand. However, the intervals of buses serving the same station were equalized, which is shown in Figure 9. When the shortturning strategy is implemented without bus holding, the stop skipping action allows buses to skip some stations and then those buses were fast to chase or overtake preceding vehicles, in which the interval standard deviation is high. However,

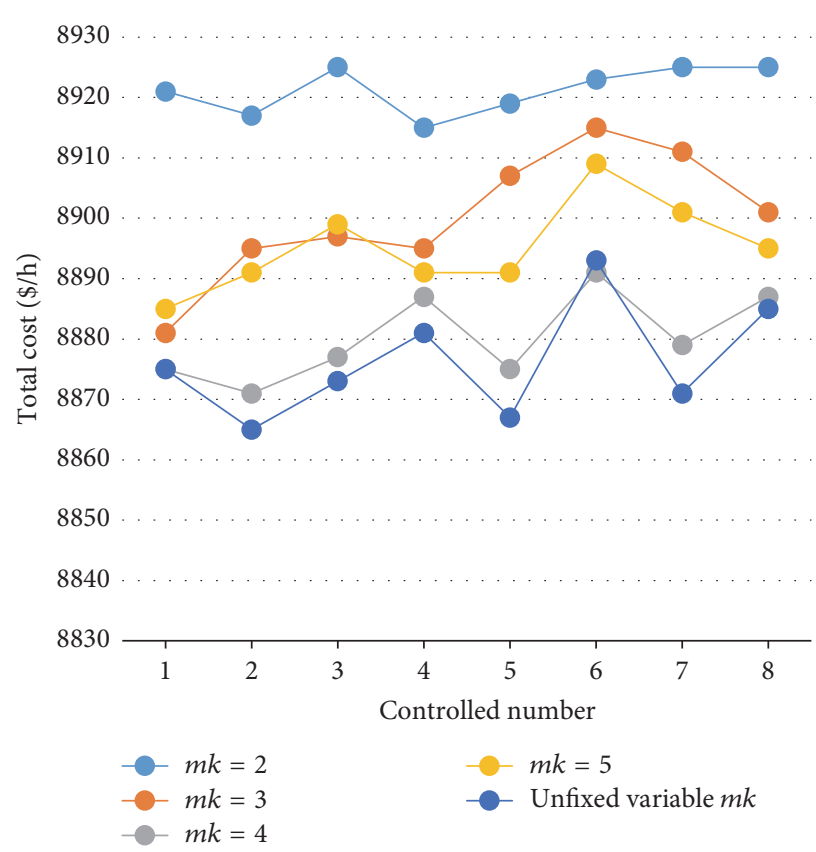

FIgURE 7: Total cost of the controlled cycle with different rolling horizons.

bus holding decreases the interval standard deviation and the intervals of buses serving the same station are equalized. Although the intervals between different segments were different, the interval standard deviation was very small, which means that passengers at the same stations can be served with the same serving time.

\section{Conclusions}

This study considered uncertain demand and investigated a dynamic short-turn controlled approach with stop skipping and bus holding actions. This approach described the two predicted discrete variables, arrival time and bus position, and used an unfixed control horizon. The actions of buses (stop skipping and bus holding) could be constantly updated. Following this strategy, buses serve some segments with high 


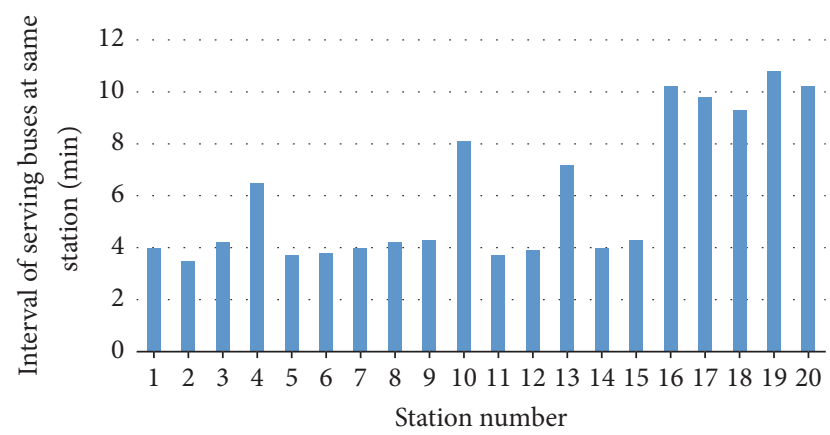

FIGURE 8: Interval of serving buses at the same station.

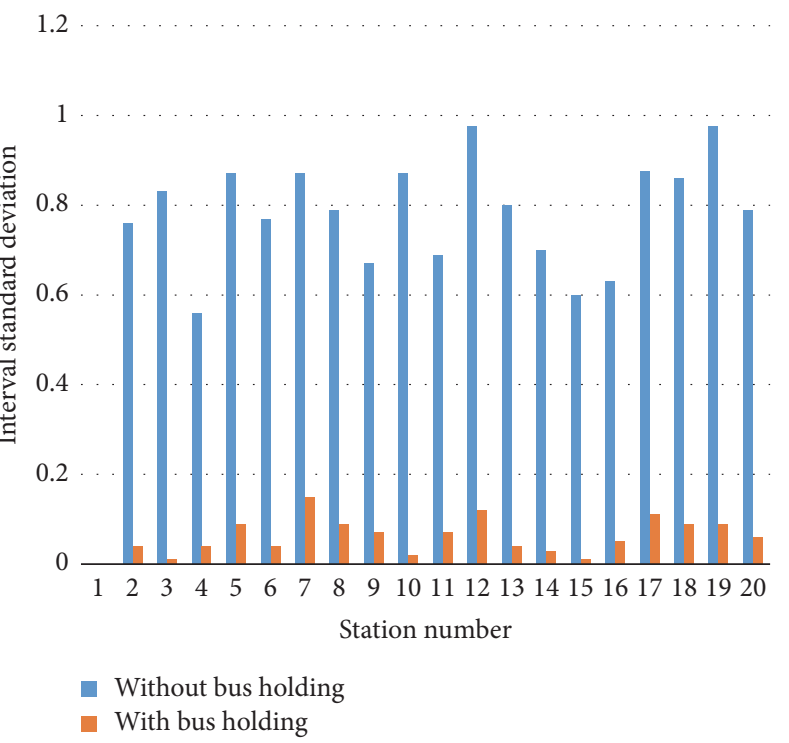

FIGURE 9: Interval standard deviations along route stations.

demand and can be held at serving stations to equalize the serving time at these stations. The optimization model aims to reduce total costs of users and operators and is solved via genetic algorithms.

The result of the test suggests that the proposed approach has a strong effect on reducing the total cost in comparison with a traditional strategy (all-stop). Furthermore, it can decrease the cost more effectively than short-turning without stop skipping or bus holding and short-turning with stop skipping, but without bus holding. Implementing an unfixed control horizon was more suitable for uncertain demand than a fixed rolling horizon and can reduce total cost more effectively. However, the improvement of transit service reliability of the stop skipping strategy is limited. Integrating bus holding and stop skipping has the capacity to equalize the serving time at the same stations.

\section{Conflicts of Interest}

The authors declare that they have no conflicts of interest.

\section{Acknowledgments}

This study was supported by the National Natural Science Foundation of China (Grant no. 51378237) and the National Science Foundation for Young Scientists of China (Grant no. 51608224).

\section{References}

[1] P. G. Furth, "Short turning on transit routes," Transportation Research Record, vol. 1108, pp. 42-52, 1987.

[2] A. Ceder, "Optimal design of transit short-turn trips," Transportation Research Record, vol. 1221, p. 22, 1989.

[3] P. Delle Site and F. Filippi, "Service optimization for bus corridors with short-turn strategies and variable vehicle size," Transportation Research Part A: Policy and Practice, vol. 32, no. 1, pp. 19-38, 1998.

[4] Y. Y. Ulusoy, S. Chien, and C.-H. Wei, "Optimal all-stop, shortturn, and express transit services under heterogeneous demand," Transportation Research Record, no. 2197, pp. 8-18, 2010.

[5] C. E. Cortés, S. Jara-Díaz, and A. Tirachini, "Integrating short turning and deadheading in the optimization of transit services," Transportation Research Part A: Policy and Practice, vol. 45, no. 5, pp. 419-434, 2011.

[6] H. Zhang, S. Zhao, H. Liu, and S. Liang, "Design of integrated limited-stop and short-turn services for a bus route," Mathematical Problems in Engineering, vol. 2016, no. 2, pp. 1-9, 2016.

[7] M. A. Turnquist and S. W. Blume, "Evaluating potential effectiveness of headway control strategies for transit systems," Transportation Research Record, no. 746, pp. 25-29, 1980.

[8] M. D. Abkowitz and M. Lepofsky, "Implementing headwaybased reliability control on transit routes," Journal of Transportation Engineering, vol. 116, no. 1, pp. 49-63, 1990.

[9] B. Yu, Z. Z. Yang, and S. Li, "Real-time partway deadheading strategy based on transit service reliability assessment," Transportation Research Part A: Policy and Practice, vol. 46, no. 8, pp. 1265-1279, 2012.

[10] J. Chen, Z. Liu, S. Zhu, and W. Wang, "Design of limited-stop bus service with capacity constraint and stochastic travel time," Transportation Research Part E: Logistics and Transportation Review, vol. 83, pp. 1-15, 2015.

[11] F. Delgado, J. C. Munoz, and R. Giesen, "How much can holding and/or limiting boarding improve transit performance?" Transportation Research Part B: Methodological, vol. 46, no. 9, pp. 1202-1217, 2012.

[12] C. E. Cortés, D. Sáez, F. Milla, A. Núñez, and M. Riquelme, "Hybrid predictive control for real-time optimization of public transport systems' operations based on evolutionary multiobjective optimization," Transportation Research Part C: Emerging Technologies, vol. 18, no. 5, pp. 757-769, 2010.

[13] D. Sáez, C. E. Cortés, F. Milla, A. Núñez, A. Tirachini, and M. Riquelme, "Hybrid predictive control strategy for a public transport system with uncertain demand," Transportmetrica, vol. 8, no. 1, pp. 61-86, 2012.

[14] Z. Liu, Y. Yan, X. Qu, and Y. Zhang, "Bus stop-skipping scheme with random travel time," Transportation Research Part C: Emerging Technologies, vol. 35, pp. 46-56, 2013. 


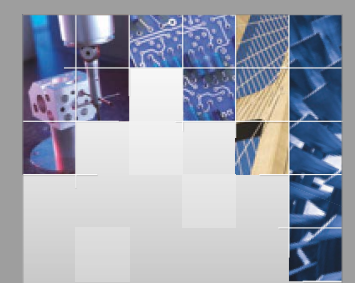

\section{Enfincering}
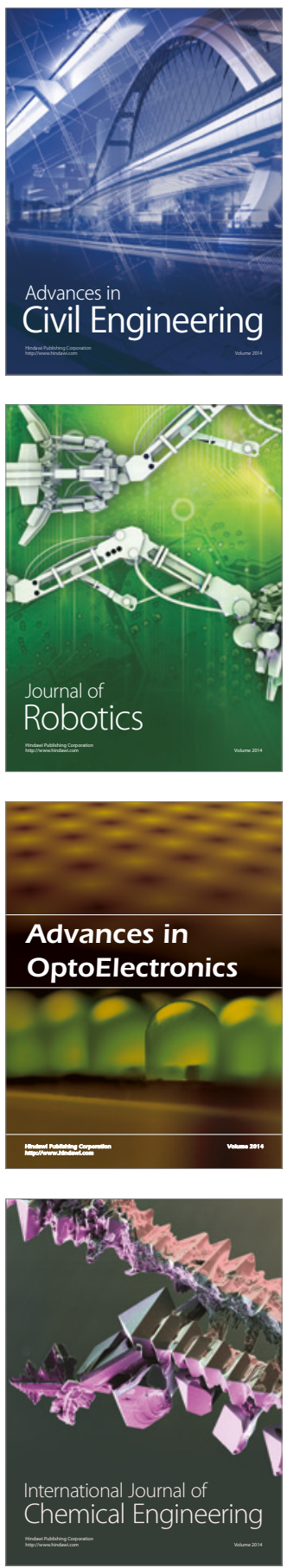

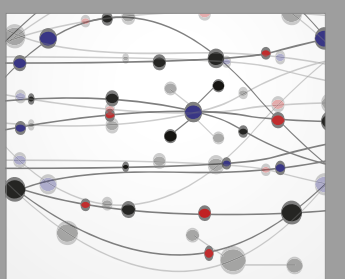

The Scientific World Journal

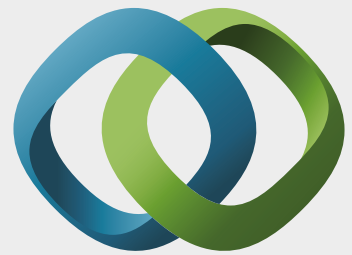

\section{Hindawi}

Submit your manuscripts at

https://www.hindawi.com
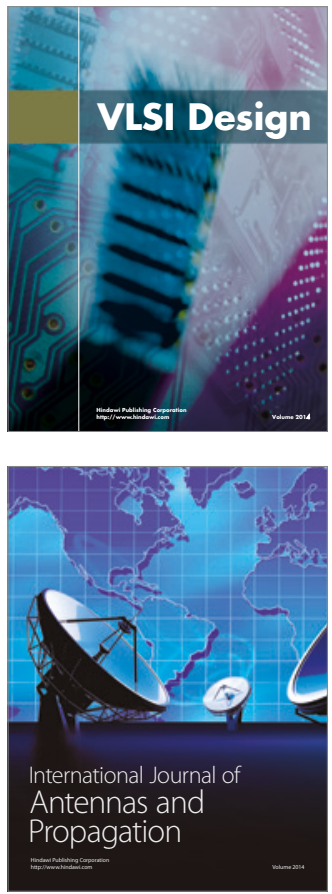

\section{Rotating}

Machinery
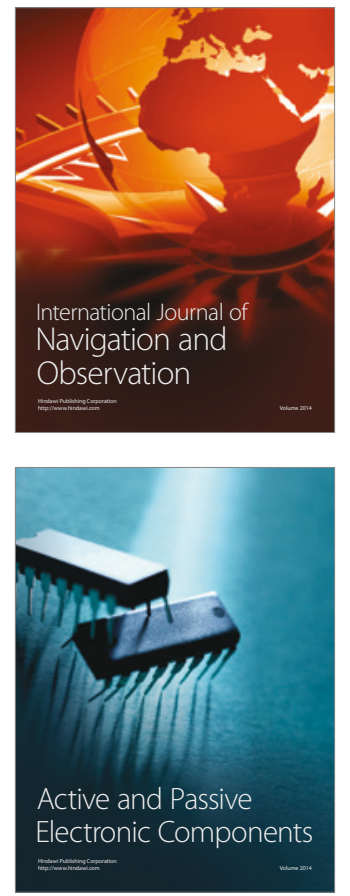
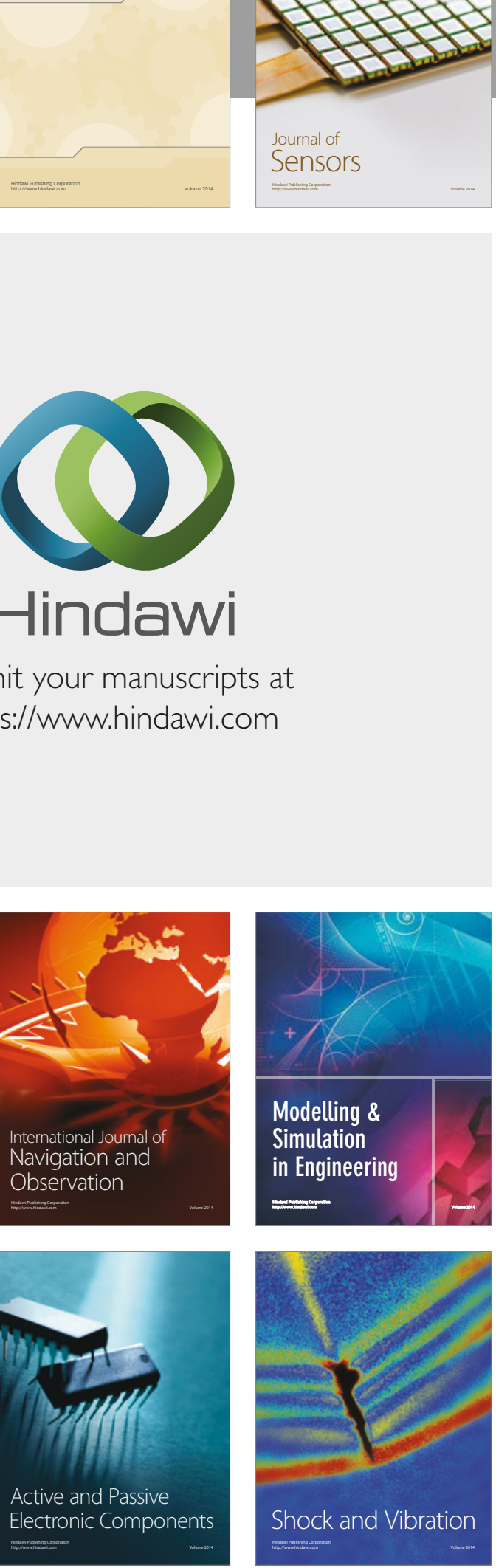
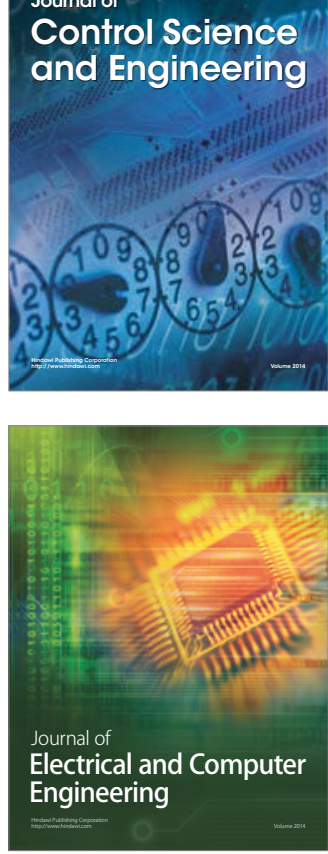

Distributed

Journal of

Control Science

and Engineering
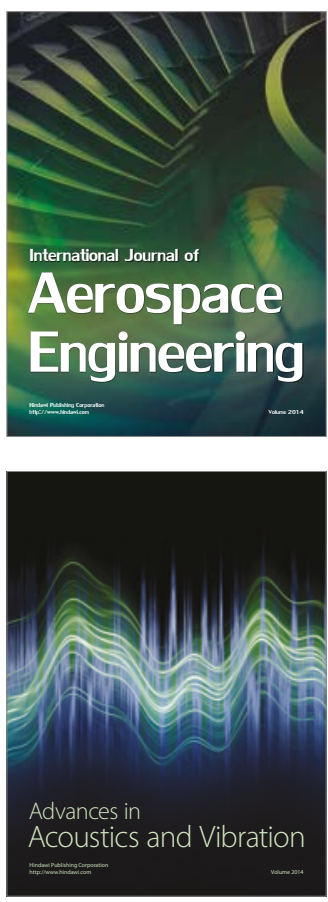

Sensor Networks 